\title{
Suppression of synchrotron radiation due to beam crystallization
}

\author{
Harel Primack* and Reinhold Blümel ${ }^{\dagger}$ \\ Fakultät für Physik, Albert-Ludwigs-Universität, Hermann-Herder-Str. 3, D-79104 Freiburg, Germany
}

(To appear in Eur. Phys. J. A, December 1998 issue)

With respect to a "hot", non-crystallized beam the synchrotron radiation of a cold crystallized beam is considerably modified. We predict suppression of synchrotron radiation emitted by a crystallized beam in a storage ring. We also propose experiments to detect this effect.

PACS: 29.20.c, 29.27.a, 41.75.i, 41.60.Ap

At the turn of the century J. J. Thomson introduced the "raisin cake" model for the structure of atoms [1]. In this model, the point-like electrons are immersed and circulate in a uniform positively-charged background. One of the interesting features of this model is the suppression of electromagnetic radiation emitted by the circulating electrons due to destructive interference. Thomson showed, for example, that six equi-spaced circulating electrons emit approximately $10^{-17}$ of the power of a single electron [2] when travelling at $1 \%$ of the speed of light. As a model for the naturally occurring atoms of the periodic table Thomson's raisin cake model was quickly replaced by the planetary models of Rutherford and Bohr. However, Thomson's model is currently experiencing a revival in the context of "artificial atoms" (see, e.g., charged particle traps [3], quantum dots [4] and clusters [5]). Moreover, present-day technology is very close to producing space-charge dominated structures that will allow us to use (and elaborate on) the ideas of Thomson.

In the context of accelerator physics, suppression of synchrotron radiation by equi-spaced charges was discussed by Schiff [6] in 1946. However, he remarks that "... it is difficult to see why the equally spaced configuration should persist".

In 1985 Schiffer and Kienle suggested the possibility of crystallized ion beams [7], i.e. geometrically-ordered structures that circulate in storage rings with speeds comparable to that of light. The spatial order is effected by the balance between Coulomb repulsion and focusing forces in the presence of strong cooling. To date there is no clear-cut experimental evidence for a fast-moving ion-beam crystal. Nevertheless, taking into account the efforts invested in such projects [8] as well as the advances in cooling techniques [9, 10], it seems likely that the goal of producing a crystalline beam will be achieved in the foreseeable future. In fact, considering the parameter regimes in which the experimentalists work, one expects that the first crystalline beams will be one-dimensional chains of equi-spaced ions, reminiscent of a scaled-up Thomson atom. Thus, we can now offer a solution to
Schiff's remark in the form of crystallized beams.

In this note we propose the use of crystalline beams (especially $1 \mathrm{D}$ chains) to achieve suppression of synchrotron radiation due to beam crystallization. In the rest of this note we shall discuss these issues and suggest experimental realizations.

The suppression of synchrotron radiation is based directly on the ideas of Thomson described above. The importance of this effect is the potential possibility to accelerate particles to very high energies such that the synchrotron radiation does not limit the final energy, even for moderately-sized accelerators. Currently, this mainly applies to electrons, since their synchrotron radiation is about $10^{13}$ larger than for protons with the same energy.

Suppose that we have $N$ particles with charge $q$ which circulate in a plane (e.g. due to a constant magnetic field) with velocity $v$ and radius of curvature $\rho$. The power emitted by a single particle is given by [11, 12]:

$$
P^{(1)}=\frac{q^{2}}{6 \pi \epsilon_{0}} \frac{\beta^{4} \gamma^{4} c}{\rho^{2}}
$$

where $\beta \equiv v / c, \gamma \equiv 1 / \sqrt{1-\beta^{2}}$, and $c$ is the velocity of light. Due to the periodicity of the motion, one can rewrite $P^{(1)}$ as:

$$
P^{(1)}=\sum_{n=1}^{\infty} P_{n}^{(1)}
$$

where $P_{n}^{(1)}$ is the contribution to the total power of radiation with frequency $\omega_{n}=n v / \rho \equiv n \omega$. It is given by [13, 14, 12]:

$$
P_{n}^{(1)}=\frac{q^{2}}{2 \pi \epsilon_{0}} \frac{c \beta}{\gamma^{2} \rho^{2}}\left[\beta^{2} \gamma^{2} n J_{2 n}^{\prime}(2 n \beta)-n^{2} \int_{0}^{\beta} J_{2 n}(2 n \xi) \mathrm{d} \xi\right] .
$$

where $J_{n}$ are the ordinary Bessel functions. For $N$ randomly placed particles, the total power emitted is simply $N$ times that of a single particle:

*email: harel@phyc1.physik.uni-freiburg.de

†email: blumel@phyc1.physik.uni-freiburg.de 


$$
P_{\text {random }}^{(N)}=N P^{(1)}=N \sum_{n=1}^{\infty} P_{n}^{(1)}
$$

For $N$ particles that are equi-spaced along the circular trajectory we have the following expression [1]:

$$
P_{\text {crystal }}^{(N)}=N^{2} \sum_{m=1}^{\infty} P_{m \cdot N}^{(1)}
$$

Thus, if $P_{n}^{(1)}$ is a rapidly decreasing function of $n$, we get an overall suppression of synchrotron radiation. Examining equation (3), this is indeed the case for all $\beta$ and for large enough $N$. To show the suppression effect in detail we plot the suppression factor $\alpha(N, \beta) \equiv P_{\text {crystal }}^{(N)} / P_{\text {random }}^{(N)}$ as a function of $N$ for $\beta \gamma=0.1,1,10$ (sub-, intermediate and ultra-relativistic regimes).
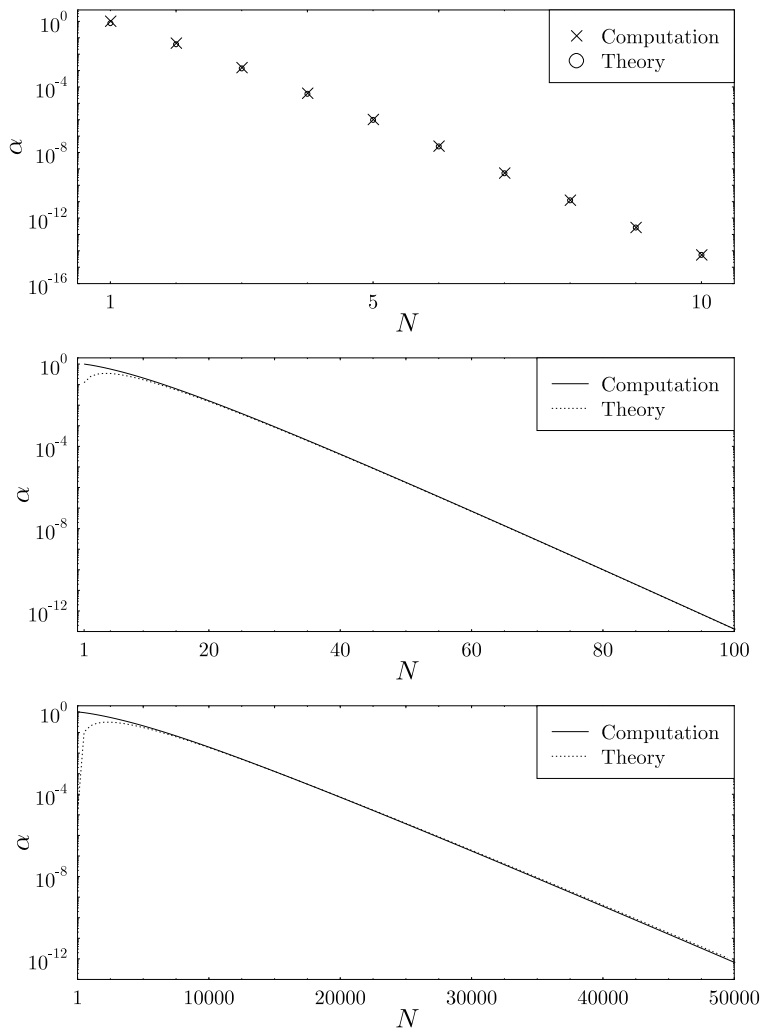

FIG. 1. Suppression factor for equi-spaced circulating particles (crystallized beam). Upper plot: $\beta \gamma=0.1$ (sub-relativistic), middle: $\beta \gamma=1$ (intermediate), lower: $\beta \gamma=10$ (ultra-relativistic). The theoretical curves correspond to equations (6, 7).

We observe in all cases exponential suppression of the synchrotron radiation for sufficiently large $N$. For subrelativistic velocities the exponential decay is manifest practically for all $N>1$. For intermediate velocities the exponential decay starts at $N \approx 10$. For ultrarelativistic velocities, the exponential decay is present for $N \gg \gamma^{3} \approx 1000$ in our case. In terms of analyti- cal expressions, one can derive the following results (e.g. [11, 12] :

$\alpha(N, \beta) \approx \frac{3 N^{\frac{3}{2}}}{4 \sqrt{\pi} \beta^{2} \gamma^{\frac{9}{2}}}\left(\frac{\beta \gamma \mathrm{e}^{\frac{1}{\gamma}}}{1+\gamma}\right)^{2 N}, \gamma \gtrsim 1, N \gg 1$,

$\alpha(N, \beta) \approx \frac{3 N^{\frac{3}{2}}}{4 \sqrt{\pi} \beta^{2} \gamma^{\frac{9}{2}}} \exp \left(-\frac{2 N}{3 \gamma^{3}}\right), \gamma \gg 1, N \gg \gamma^{3}$.

The analytical formulas clearly fit the numerical computations in their range of validity.

At currently achievable magnetic fields even ultrarelativistic ion beams emit very little synchrotron radiation power. Hence, the experimental observation of the suppression effect for ions is very difficult. However, for ultra-relativistic electrons the synchrotron radiation power is appreciable. Thus, our theoretical calculations may motivate experiments on crystallization of electron beams. In order to experimentally observe the suppression effect, we suggest two types of experiments:

1. Start from cold electrons at rest which form a circular chain, and accelerate them such that the crystalline order is maintained. The initial crystallization may be realized by resistive cooling techniques [15.

2. Accelerate electrons and apply sympathetic cooling by a fast crystallized ion beam. This is the inverse mechanism of electron cooling that is used currently in storage rings $[8$. Sufficiently strong cooling of the electron beam leads to its crystallization, and hence to dramatic suppression of synchrotron radiation.

Further work is needed in order to evaluate the feasibility of the suggested experiments.

HP acknowledges a MINERVA scholarship. RB acknowledges financial support from the Deutsche Forschungsgemeinschaft (SFB 276).

[1] J. J. Thomson, Die Korpuskulartheorie der Materie. (Vieweg, Braunschweig, 1908).

[2] J. J. Thomson, Elektrizität und Materie. (Vieweg, Braunschweig, 1909).

[3] R. Blümel, Bull. Am. Phys. Soc. 39, 1177 (1994).

[4] M. A. Kastner, Physics Today 46(1), 24 (1993).

[5] H. Haberland, Clusters of atoms and molecules. (Springer, Berlin, 1994).

[6] L. I. Schiff, Rev. Sci. Inst. 17, 6 (1946).

[7] J. P. Schiffer and P. Kienle, Z. Phys. A 321, 181 (1985).

[8] D. Habs and R. Grimm, Ann. Rev. Nucl. Part. Sci. 45, 391 (1995).

[9] J. S. Hangst, M. Kristensen, J. S. Nielsen, O. Poulsen, J. P. Schiffer, P. Shi, Phys. Rev. Lett. 67, 1238 (1991). 
[10] H.-J. Miesner, R. Grimm, M. Grieser, D. Habs, D. Schwalm, B. Wanner, and A. Wolf, Phys. Rev. Lett. 77, 623 (1996).

[11] J. D. Jackson, Classical Electrodynamics, 2nd edition. (John Wiley \& Sons, New York, 1975).

[12] L. M. Landau and E. M. Lifshitz, The classical theory of fields, 4th edition. (Pergamon Press, Oxford, 1979).
[13] G. A. Schott, Electromagnetic Radiation. (Cambridge University Press, Cambridge, 1912).

[14] A. A. Kolomensky and A. N. Lebedev, Theory of Cyclic Accelerators. (North-Holland Publishing Company, Amsterdam, 1966).

[15] Ghosh, Ion Traps. (Clarendon Press, Oxford, 1995). 